\title{
Influence of Characteristics of Self- actualization and Coping Behavior on Resistance of Teachers to Professional Stressors and Emotional Burnout
}

\author{
Elena Voitenko*, Hryhorii Kaposloz, Serhii Myronets, Oksana Zazymko, Vasyll Osodlo
}

Kyiv National University of Trade and Economics, Ukraine

Keywords:

Professional Stress,

Emotional Burnout, Coping

Behavior, Coping Strategy,

Self-actualization

Received

20 November 2020

Received in revised form

30 November 2020

Accepted

02 December 2020

*Correspondence:

evoytenko73@gmail.com

\begin{abstract}
The availability of conflicting data in the scientific literature on the correlations between selfactualization, occupational stress and emotional burnout has suggested that the nature of the relationship between occupational stress and self-actualization and emotional burnout may change due to some other variables, such as coping strategies. The aim of the study is to determine the nature of the relationship between emotional burnout as a result of occupational stress with the characteristics of self-actualization and the peculiarities of coping behavior of teachers. In a pilot study organized by the cross-sectional design, a survey of 107 Ukrainian teachers was conducted. According to the purpose of the study, the following diagnostic techniques were used: Victor Boyko's method of diagnosing the level of emotional burnout, version of the Russian-language adaptation of Shostrom's self-actualization test SAT and Ways of Coping Questionnaire. The main hypotheses of this study were tested using correlation analysis and analysis of variance. It was found that the severity of burnout is negatively correlated with self-actualization. In addition, the joint influence of selfactualization indicators and certain coping strategies on the nature of the formation of different stages of emotional burnout was detected. It was also determined that coping strategies are formed under the influence of two factors. On the one side, it is professional stress and burnout, and on the other side, it is the desire to satisfy the need for self-actualization. The results showed that the predictor of successful adaptation of teachers to the requirements of professional activity are certain characteristics of the personal profile of coping behavior, which have a mitigating effect on stress factors determined by self-actualization. Future research is needed to determine the effectiveness of certain coping strategies on teachers' resistance to occupational stressors and burnout over a longer period of follow-up.
\end{abstract}


The problem of emotional burnout of social workers is not new in psychology, but the search for effective ways to prevent this syndrome is still ongoing. Factors that help to cope with the negative effects of occupational stress and prevent emotional burnout include the teacher's personal potential in terms of the level of development of needs for self-actualisation, ability to fully identify and develop their capabilities and personal set of ways to overcome obstacles in various areas of mental activity. Some authors consider the process of self-actualization of the individual as a systemforming personal resource to overcome burnout (Babich, 2007), others as a factor of resistance to burnout (Kurapova, 2009). Coping behavior is highlighted as a major variable that affects the ability to resist the negative effects of occupational stressors (Lazarus \& Folkman, 1984). Data on the correlations between the level of self-actualization, occupational stress and emotional burnout in different studies are different (Bohan, 2008; Kurapova, 2009). These discrepancies suggest that the nature of the relationship between occupational stress, self-actualization, and emotional burnout may change due to the influence of certain other variables, such as certain coping strategies. Therefore, the main purpose of this study is to examine the nature of the relationship between selfactualization, occupational stress and emotional burnout, taking into account the mediating effect of coping behavior.

\section{Literature Review}

A review of the literature showed an active study of various ways and mechanisms of human adaptation to the requirements of professional activity, which is directly related to the issue of stress. It is shown that emotional burnout occurs due to chronic stress as a "syndrome of physical and emotional exhaustion" (Maslach, 2003). Occupational burnout occurs against the background of the stress of interpersonal interaction, but its appearance indicates that the individual is prone to other stressors. Professionals, who are characterized by the presence of emotional burnout, in most cases are not able to overcome the emotional stress associated with the work (Maslach, 2003). Researchers note the mediating role of social support in the relationship between role stress and job satisfaction (de Almeida, 2019), the effects of emotional intelligence, self-efficacy and psychological empowerment on psychological exhaustion and job satisfaction (Sattari Ardabili, 2020), the role of internal determination in the regulation of the state of the professional (Skaalvik \& Skaalvik, 2015; Voitenko, 2020). It is shown that different individuals react differently to the same stressor. The type of response and direction of behavior depends on the psychological characteristics of individuals and, in particular, on their individual psychological resources of coping.

Individual psychological factors that determine the development of stress were first analyzed by R. Lazarus. Central to his theory is the subjective significance of the situation in which a person acts and the behavioral strategies by which this person tries to overcome difficulties (Lazarus, 1974). To indicate a set of cognitive and behavioral methods used by an individual to reduce the effects of stress, Lazarus and Folkman (1984) introduced the concept of "coping". Sustainable forms of coping behavior are called coping strategies (Lazarus, 2006). Problem-oriented coping strategies are aimed at overcoming the source of stress, emotionally-oriented - to overcome the emotional arousal caused by stress. Both types of coping are more often considered in the context of emotional regulation. The psychological purpose of coping is to adapt a person to the requirements of the situation, the ability to master it, weaken or mitigate the requirements and thus neutralize the stress effect of the situation (Losoya, Eisenberg, Fabes, 1998). So, it can be assumed 
that certain coping strategies can slow down the development of emotional burnout, reducing the negative impact of occupational stressors.

Recently, a resource approach has emerged in the study of coping strategies. According to this approach coping behavior is implemented on the basis of coping resources, which determine the characteristics of the interaction of personality and social environment, facilitate adaptation to life situations. The resource approach emphasizes the process of resource allocation, so that some people are able to maintain health and adapt, despite different life circumstances (Frydenberg, 2002). Proponents of this approach consider a wide range of different resources: the availability of emotional help from the social environment (Aripova, 2019), skills and abilities of the individual (Bagheri et al., 2019). One of the most significant system-forming personal resources is selfactualization (Kurapova, 2009).

There is conflicting data in the current scientific literature regarding the nature of the relationship between self-actualization, occupational stress, and burnout. On the one side, self-actualization is characterized by productivity and satisfaction, growth of mental and personal activity, the transition to a qualitatively new level of activity (Babich, 2007). It is shown that self-actualization is one of the most important supporting resources of the individual to overcome burnout. Burnout syndrome has negative correlations with indicators of self-actualization in pedagogical activities (Nalichaeva, 2010). On the other side, there is data that the subjective image of professional development and self-realization of the individual, personal ambitions can also be determinants of professional stress, which is a complex phenomenon of causality, and arises not only in response to difficulties and special demands of the profession. Self-realization of a person as a way of his existence as an open system can also cause stress, because it requires lifestyle adjustments, changes in perceptions of the world, etc., which sometimes situationally deprives a person of resources. A person who is unable to exercise their capabilities is doomed to chronic and destructive stress (Bohan, 2008). The theoretical analysis and the identified contradictions allow us to formulate a theoretical hypothesis: the mediator between self-actualization as a determinant of occupational stress and emotional burnout are certain coping behavior strategies that mitigate the negative effects of stressors and promote resistance to burnout.

Figure 1 shows the theoretical basis of our study. In this model, self-actualization mediates the negative effects of occupational stress and slows the development of emotional burnout. On the other side, self-actualization can provoke stress, and the relationship between these variables is mediated by coping behavior. That is to say, the level of emotional burnout as a result of occupational stress varies depending on the nature of coping behavior.

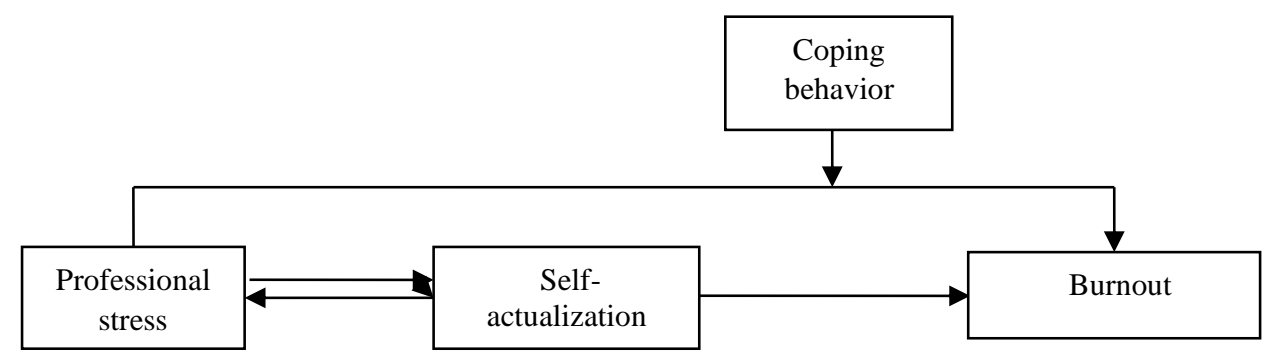

Figure 1. Theoretical basis of the study 


\section{Method}

A pilot study for determining the presence and nature of the relationship between coping, selfactualization and emotional burnout as a result of professional stress in pedagogical activity was organized using the cross-sectional method. The research program was based on an experimental hypothesis: the nature of the relationship between emotional burnout as a result of occupational stress and self-actualization can change due to the influence of some other variables, including coping behavior strategies. The participants of the study were 107 Ukrainian teachers of higher education, selected by random sampling technique. This sample size is sufficient for a pilot study aimed at identifying the main trends in the relationship between selected variables. The sociodemographic characteristics of respondents are presented in Table 1.

Table 1

Demographic Characteristics of Respondents

\begin{tabular}{lll}
\hline & & $\%$ \\
\hline Age & $25-35 \mathrm{yrs}$ & 18.7 \\
& $36-45 \mathrm{yrs}$ & 27.1 \\
& $46-55 \mathrm{yrs}$ & 46.7 \\
Experience & $>56 \mathrm{yrs}$ & 7.5 \\
& $1-10 \mathrm{yrs}$ & 24.3 \\
& $11-20 \mathrm{yrs}$ & 30.8 \\
Gender & $21-30 \mathrm{yrs}$ & 34.6 \\
& $>30 \mathrm{yrs}$ & 10.3 \\
\hline
\end{tabular}

According to the purpose of the study, the following diagnostic tools were used: Victor Boyko's (Boyko, 1996) method of diagnosing the level of emotional burnout, version of the Russianlanguage adaptation of Shostrom's self-actualization test SAT (Fetiskin, Kozlov, \& Manuilov, 2002) and Ways of Coping Questionnaire by R. Lazarus and S. Folkman (Bityutskaya, 2015).

Questionnaire "Diagnosis of the level of emotional burnout", developed and standardized by Boyko (1996), was used to identify the level of development of emotional burnout and its individual components. The tool allows to identify the main symptoms of emotional burnout, which make up the three phases of its formation. The phase of tension is the experience of traumatic circumstances, self-dissatisfaction, anxiety and depression. The phase of resistance is inadequate selective emotional response, emotional and moral disorientation, expansion of the sphere of saving emotions, reduction of professional responsibilities. The phase of exhaustion is emotional deficit and alienation, depersonalization, psychosomatic and psychovegetative disorders. The method is to fill out a questionnaire of 84 items, each question involves a clear answer "yes" or "no". The questions are grouped into three scales, which allows to diagnose the formation of each phase. Each scale is divided into four subscales that represent the corresponding symptoms. The total scores obtained for each of these subscales allow us to judge the level of formation of each phase and varies in the range from 0 to 120 (36 points or less - phase has not developed; 37-60 points - phase is developing; 61 points or more - phase had developed).

The Ways of Coping Questionnaire (WCQ) developed by Lazarus and Folkman (1984), was used to diagnose coping strategies. Russian-language adaptation of the questionnaire was developed and standardized by Bityutskaya (2015). The questionnaire was specifically designed to measure coping with stress in specific situations. It consists of eight subscales, which include coping techniques such as confrontation, distancing, self-control, seeking social support, taking responsibility, avoiding, solving problems, and positively reassessing the situation. The processing 
of the results is the summation of points for each scale and then their calculation according to the formula: $X=$ sum of points / $\max$ score $* 100$. The questionnaire offers 50 items and five answer options: never - 0 points; rarely - 1 point; sometimes - 2 points; often - 3 points.

E. Shostrom's questionnaire was used to assess self-actualization indicators. A version of the Russian-language adaptation of the Shostrom self-actualization test was developed and standardized by Yu. Alyoshina, L. Gozman, M. Zagika and M. Croz at the Department of Social Psychology of Moscow State University (Fetiskin et al., 2002). The self-actualization test (SAT) measures self-actualization on two independent base scales (Time orientation and Support) and twelve additional ones (value orientation, flexibility of behavior, sensitivity to yourself, spontaneity, self-esteem, self-acceptance, representation of human nature, synergy, acceptance of aggression, contact, cognitive needs, creativity). Questionnaire scales characterize the main areas of self-actualization. Additional scales, in contrast to the basic ones, which measure the global characteristics of self-actualization, are focused on the registration of its individual aspects. The questionnaire has 125 items. Each answer that matches the key is worth 1 point. The processing of the results is the summation of points for each scale. The practice of using SAT in various studies is based on the following scale estimates: 55-70 T-points - overestimated values, which indicate the influence of the factor of social desirability on the results; 45-55 T-points - mental and statistical norm; 40-45 T-points and less is a range typical for various forms of mental disorders. SAT as a research method allows also the using of "raw" scores.

The main hypotheses of this study were tested using correlation analysis and analysis of variance. Data processing was carried out using a program IBM SPSS Statistics 22. Proving the experimental hypothesis requires answers to the following questions:

1. What are the features of relationship between coping and self-actualization and emotional burnout as a result of occupational stress?

2. How do indicators of coping behavior interact with indicators of self-actualization?

3. How is the duration of influence of occupational stressors related to the symptoms of emotional burnout, the formation of certain coping strategies and the level of self-actualization of teachers?

The null and alternative hypotheses were formulated to statistically confirm the relationship between indicators of coping, self-actualization and emotional burnout as a result of occupational stress.

Ho: There are no significant differences between indicators of coping and self-actualization in groups of teachers with different levels of emotional burnout.

H1: There are significant differences between the indicators of coping and self-actualization in groups of teachers with different levels of emotional burnout.

Acceptance or rejection of these hypotheses was carried out using the procedure of analysis of variance. The correctness of the application of analysis of variance to the scales of all selected questionnaires was checked using the Homogeneity of variance test Levene. The Brown-Forsythe criterion for the validity of conclusions about differences between means was applied in the case of discrepancy. To statistically confirm the existence of relations between indicators of coping behavior and self-actualization, two hypotheses were also formulated:

Ho: Indicators of the level of preference for certain coping strategies do not correlate with the level of self-actualization.

H1: Indicators of the level of preference for certain coping strategies correlate with the level of self-actualization. 
Acceptance or rejection of these hypotheses was carried out using the procedure of correlation analysis. The correctness of the application of Spearman correlation analysis is conditioned by the affiliation of all scales of the selected methods to the ordinal, and the inconsistency of the obtained frequency distribution values to the normal distribution. To statistically confirm the relationship between the influence of occupational stress factors, the manifestation of symptoms of emotional burnout, the formation of coping strategies and various aspects of self-actualization of teachers, two hypotheses were formulated:

$\mathbf{H}_{\mathbf{0}}$ : Indicators of duration of occupational stress factors (experience, age) do not correlate with the phases of emotional burnout (tension, resistance, exhaustion), the formation of coping strategies and self-actualization.

H1: Indicators of duration of occupational stress factors (experience, age) correlate with the phases of emotional burnout (tension, resistance, exhaustion), the formation of coping strategies and self-actualization.

Acceptance or rejection of these hypotheses was carried out using the procedure of correlation analysis. The correctness of the application of Spearman correlation analysis is conditioned by the affiliation of all scales of the selected methods to the ordinal, and the inconsistency of the obtained frequency distribution values to the normal distribution.

\section{Results}

Significant differences between the indicators of the level of self-actualization in groups of teachers with low (1), medium (2), high (3) levels of emotional burnout phases "Tension", "Resistance", "Exhaustion" according to the Boyko`s questionnaire are presented in Table 2. Analysis of the nature of the connections proved by significant differences between the indicators of the level of self-actualization in groups of teachers with low (1), medium (2), high (3) levels of emotional burnout phases "Tension", "Resistance", "Exhaustion" (Table 2 ) allows us to identify two trends that confirm the statistical hypothesis, but have different characteristics of the manifestation of differences.

Table 2

Influence of Indicators of Self-actualization on Phases of Emotional Burnout of Teachers

\begin{tabular}{|c|c|c|c|c|c|c|c|c|c|}
\hline \multirow{2}{*}{$\begin{array}{l}\text { Burnout phases } \\
\text { Scales }\end{array}$} & \multicolumn{3}{|c|}{ Tension } & \multicolumn{3}{|c|}{ Resistance } & \multicolumn{3}{|c|}{ Exhaustion } \\
\hline & $\mathrm{F}$ & MSE & $\mathrm{p}$ & $\mathrm{F}$ & MSE & $\mathrm{p}$ & $\mathrm{F}$ & MSE & $\mathrm{p}$ \\
\hline Support & 7.30 & 49.78 & $\leq .001$ & - & - & - & - & - & - \\
\hline Time orientation & 4.18 & 48.76 & $\leq .02$ & 4.173 & 48.76 & $\leq .02$ & - & - & - \\
\hline Value orientation & 24.98 & 47.43 & $\leq .001$ & 10.604 & 47.43 & $\leq .001$ & 10.17 & 47.43 & $\leq .001$ \\
\hline Flexibility of behavior & 12.73 & 47.15 & $\leq .001$ & - & - & - & 4.02 & 47.15 & $\leq .05$ \\
\hline Sensitivity to yourself & 4.91 & 49.14 & $\leq .01$ & - & - & - & 4.49 & 49.14 & $\leq .02$ \\
\hline Spontaneity & 15.12 & 50.41 & $\leq .001$ & 2.862 & 50.41 & $\leq .05$ & 7.00 & 50.41 & $\leq .001$ \\
\hline Self-esteem & 10.08 & 53.11 & $\leq .001$ & - & - & - & - & - & - \\
\hline Self-acceptance & - & - & - & - & - & - & - & - & - \\
\hline $\begin{array}{l}\text { Representation about } \\
\text { human nature }\end{array}$ & 6.93 & 51.22 & $\leq .001$ & - & - & - & 9.82 & 51.22 & $\leq .001$ \\
\hline Synergy & 8.93 & 47.38 & $\leq .001$ & - & - & - & 10.89 & 47.38 & $\leq .001$ \\
\hline $\begin{array}{l}\text { Acceptance of } \\
\text { aggression }\end{array}$ & - & - & - & - & - & - & - & - & - \\
\hline Contact & - & - & - & - & - & - & - & - & - \\
\hline Cognitive needs & 9.06 & 42.87 & $\leq .001$ & - & - & - & 5.18 & 42.87 & $\leq .01$ \\
\hline Creativity & - & - & - & - & - & - & - & - & - \\
\hline
\end{tabular}

Note. Support" (significant differences were found between low and medium levels $(* * p<.01)$, 
First, in groups of teachers with different levels of emotional burnout, significant differences were found between indicators of self-actualization, which proves an alternative statistical hypothesis. The linear nature of these differences indicates that a high level of self-actualization prevents the development of the tension phase. This trend is confirmed by the distribution of indicators of self-actualization in groups of teachers with low (1), medium (2), high (3) levels of the phase "Tension" on the following scales (Figure 2):

- "Support" (significant differences were found between low and medium levels $(* * p<.01)$, between low and high levels $\left({ }^{*} p<.05\right)$ (Figure $2 \mathrm{a}$ );

- "Time orientation" (significant differences were found between low and high levels $\left({ }^{*} p\right.$ $<.05$ ) (Figure 2b);

- "Flexibility of behavior" (significant differences were found between low and medium levels $(* * * p<.001)$, between low and high levels $(* * * p<.001)$, and between medium and high levels $(* p<.05)$ (Figure $2 c)$; "Sensitivity to self" (significant differences were found between low and medium levels $(* p<.05)$ (Figure $2 \mathrm{~d}$ );

- "Spontaneity" (significant differences were found between low and medium levels $(* * * p$ $<.001)$, between low and high levels $(* * p<.01)$ (Figure 2e);

- "Representation about human nature" (significant differences were found between low and medium levels $(* * p<.01)$, between low and high levels $(* p<.05)$ (Figure $2 \mathrm{f})$;

- "Synergy" (significant differences were found between low and medium levels $(* * p<.01$ ), between low and high levels $(* p<.05)$ (Figure $2 \mathrm{~g}$ );

- "Cognitive needs" (significant differences were found between low and medium levels $(* * * p<.001)$, between low and high levels $(* p<.05)$ (Figure $2 \mathrm{~h})$. 


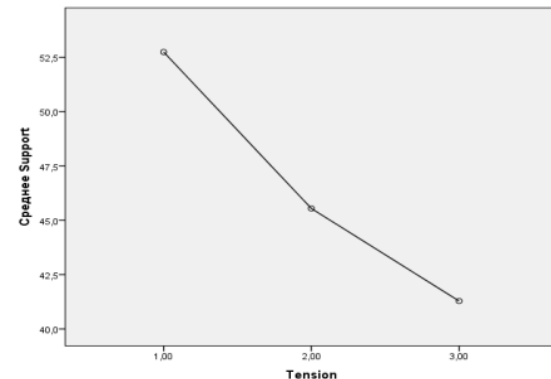

a)

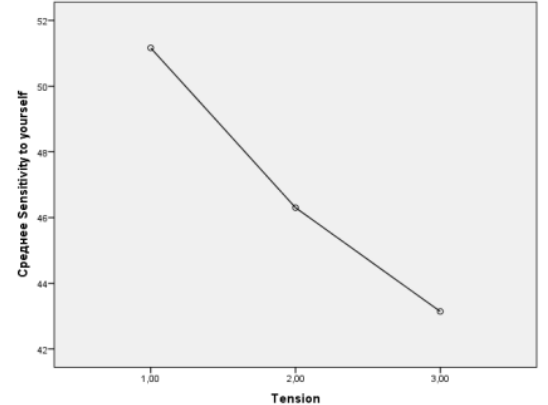

d)

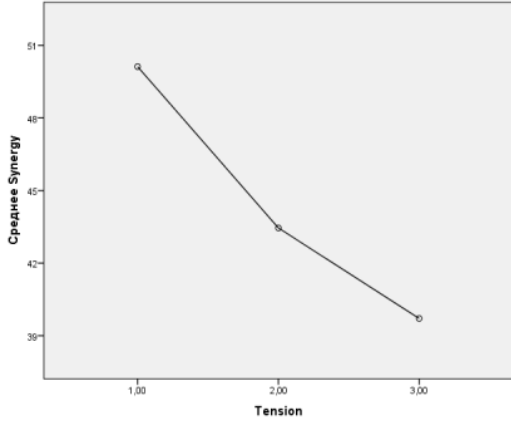

g)

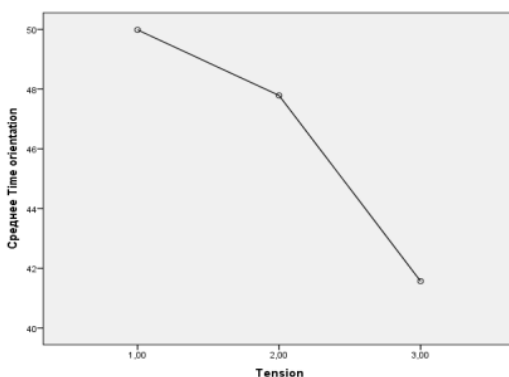

b)

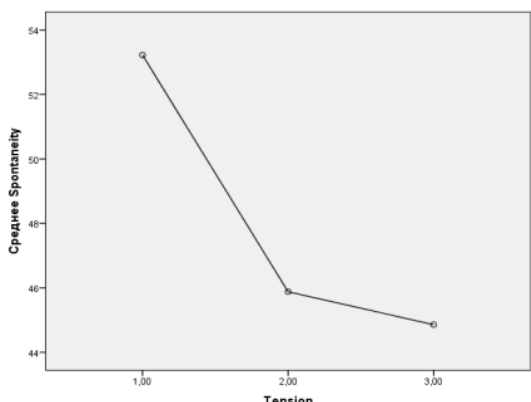

e)

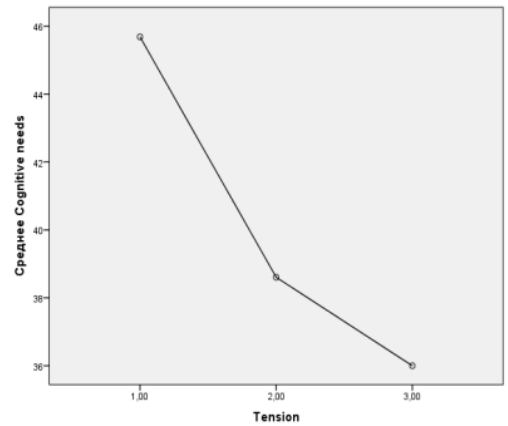

h)

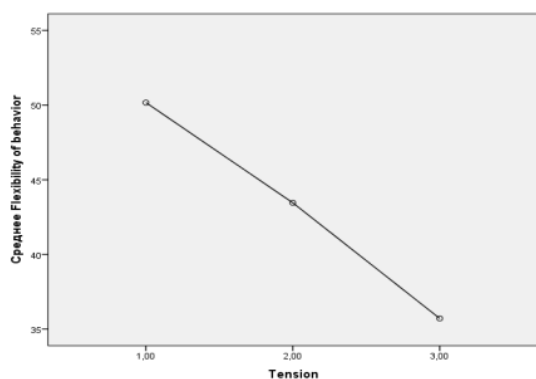

c)

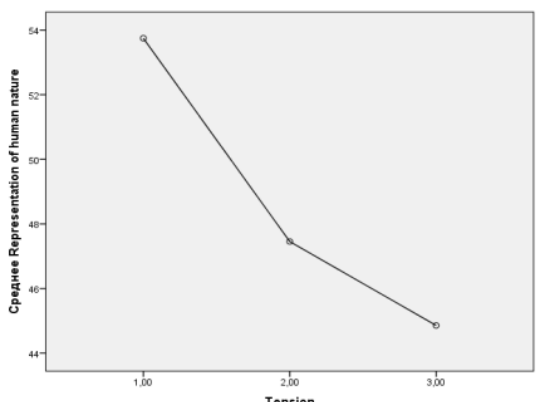

f)

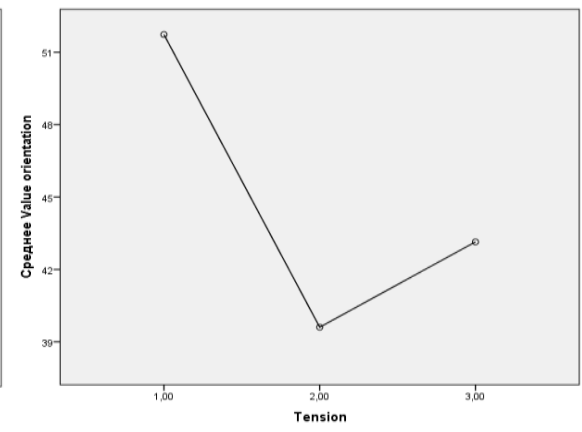

i)

Figure 2. Differences between indicators of self-actualization in groups of teachers with low (1), medium (2), high (3) levels of the phase of emotional burnout "Tension"

Dynamics of change in the average values of self-actualization on the scale "Value Orientation" in groups of teachers with low (1), medium (2) and high (3) levels of the phase of emotional burnout "Tension" has no pronounced of linear descending nature (Figure 2i). Nevertheless, the nature of the differences confirms the first trend, as significant differences were found between indicators of self-actualization in groups of teachers with low and medium $(* * * p<.001)$, low and high $(* p<$ .05) levels of emotional burnout. Significant differences between the indicators of self-actualization on the scale "Value Orientation" were not found in groups of teachers with medium and high levels of the phase of emotional burnout "Tension".

The first trend is also confirmed by the distribution of indicators of self-actualization in groups of teachers with low (1), medium (2) and high (3) levels of the phase of emotional burnout "Resistance" on the following scales. The nature of the differences is illustrated through the graphs in Figure 3. Regarding "Spontaneity", significant differences were found between low and high levels (* $*^{*}$.05) (Figure 3a); and "Value orientation", significant differences were found between low and medium levels $(* * * p<.001)$, between low and high levels $(* * * p<.001)$ (Figure $3 b$ ). 


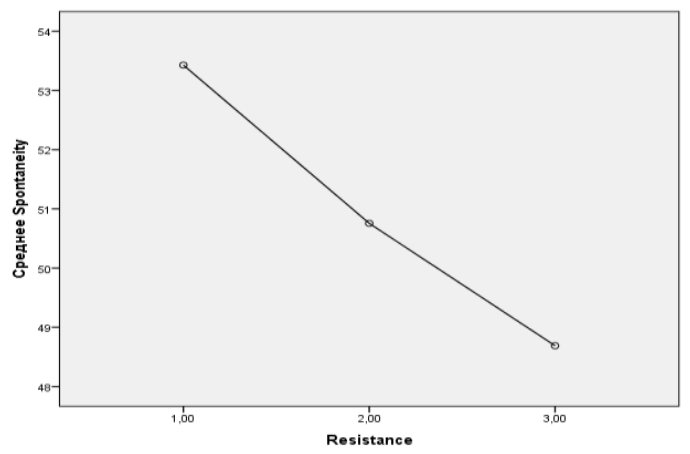

a)

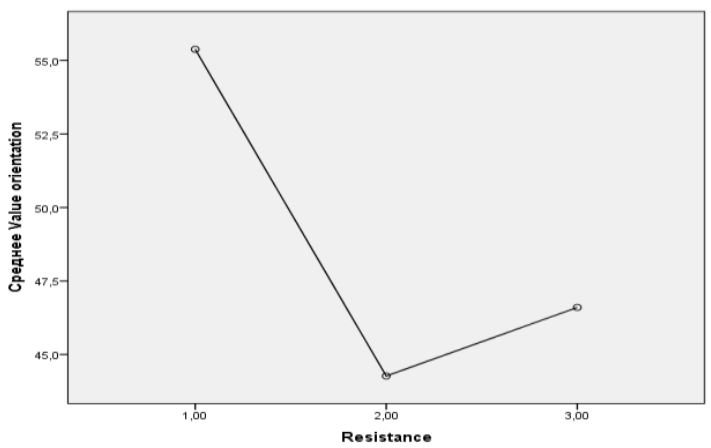

b)

Figure 3. Differences between indicators of self-actualization in groups of teachers with low (1), medium (2), high (3) levels of the phase of emotional burnout "Resistance"

The distribution of indicators of self-actualization on certain scales in groups of teachers with low (1), medium (2), high (3) levels of manifestation of the phase of emotional burnout "Exhaustion" also confirms the first trend. The nature of the differences is illustrated through the graphs in Figure 4.

Regarding "Value orientation", significant differences were found between low and medium levels $(* * * p<.001)$, between low and high levels $(* * p<.01)$ (Figure $4 \mathrm{a})$; "Flexibility of behavior", significant differences were found between low and medium levels $(* * p<.01)$ (Figure $4 \mathrm{~b}$ ); "Synergy", significant differences were found between low and medium levels $(* * * p<.001)$, between low and high levels $(* * p<.01)$ (Figure $4 c)$; "Cognitive needs", significant differences were found between low and medium levels (* < .05) (Figure 4d); "Sensitivity to yourself", significant differences were found between low and medium levels $\left({ }^{*} p<.05\right)$ (Figure $4 \mathrm{e}$ ); and "Spontaneity", significant differences were found between low and medium levels $(* * p<.01)$ (Figure 4f). The decline of indicators in the Sensitivity to self and Spontaneity scales is not gradual. They increase slightly at the third (high) level of the "Exhaustion" phase. However, this increase is not statistically significant. 


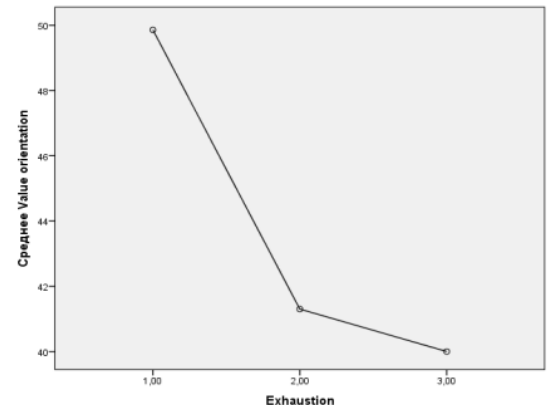

a)

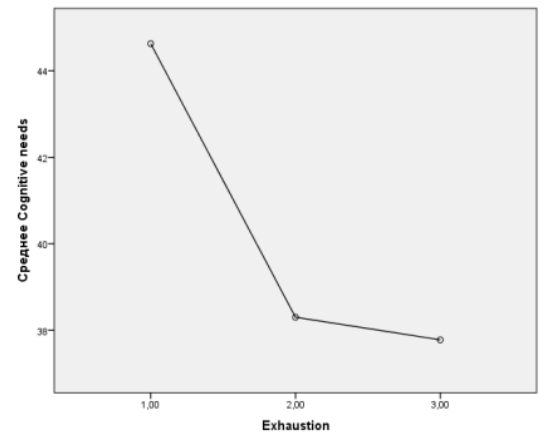

d)

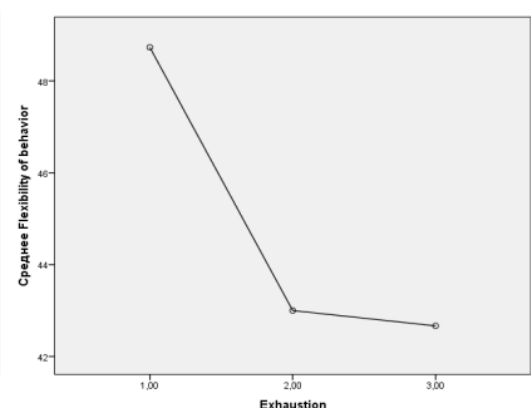

b)

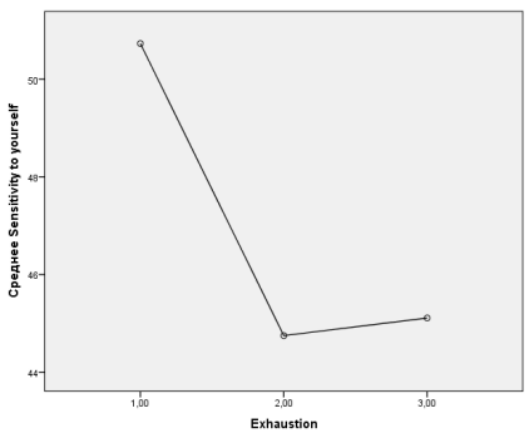

e)

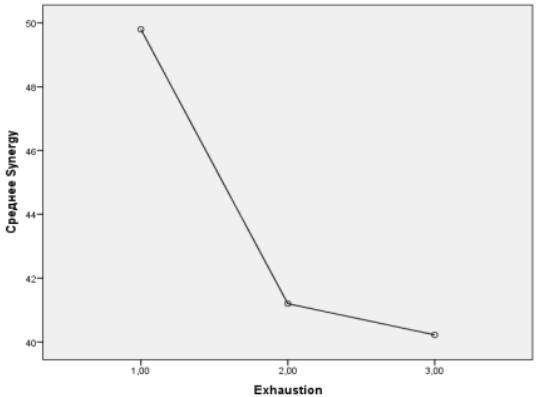

c)

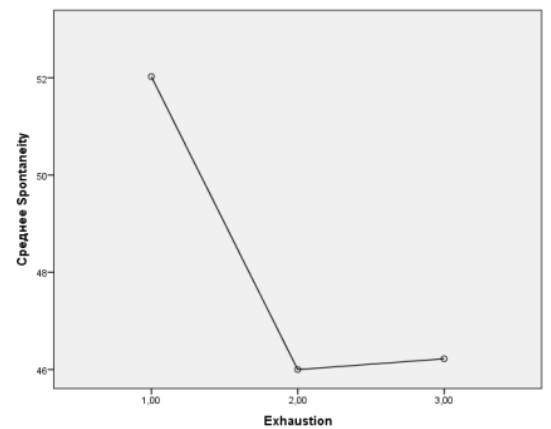

f)

Figure 4. Differences between indicators of self-actualization in groups of teachers with low (1), medium (2), high (3) levels of the phase of emotional burnout " Exhaustion"

Second, significant differences were found between indicators of self-actualization in groups of teachers with different levels of emotional burnout, which proves the alternative statistical hypothesis. The differences are not linear. This trend is illustrated by the following graphs in Figure 5.

The second trend is confirmed by the distribution of average values of indicators of selfactualization on the scale Self-esteem in teachers with low (1), medium (2), high (3) levels of the phase of emotional burnout "Tension". According to Tukey's criterion, two homogeneous subgroups for alpha .05 were identified. The first group combines medium and high levels of the Tension phase; the second group combines high and low levels of the phase of Tension. That is, a high level, not a medium one, is intermediate between the other two tension levels (Figure 5a).

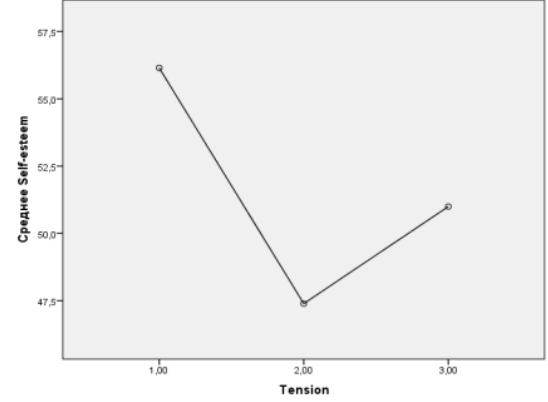

a)

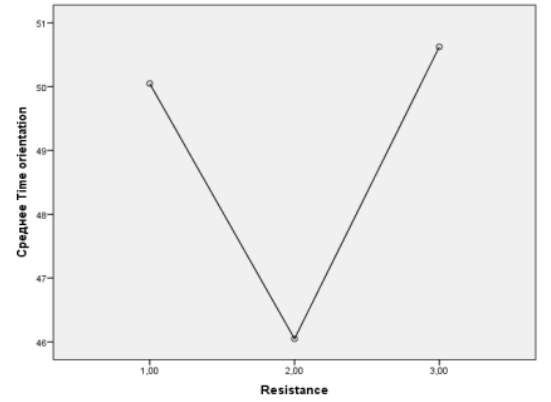

b)

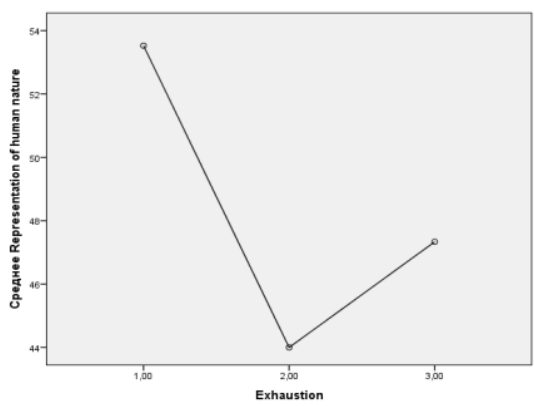

c)

Figure 5. Differences between indicators of self-actualization in groups of teachers with low (1), medium (2), high (3) levels of emotional burnout, which prove the alternative statistical hypothesis

The second trend is also confirmed by the distribution of indicators of self-actualization in groups of teachers with low (1), medium (2), high (3) levels of the phase of emotional burnout "Resistance" on the scale "Orientation in time". Significant differences were found between 
medium and high levels $(* p<.05)$. In this case, high level of self-actualization on the scale "Orientation in time" are observed in teachers with both low and high levels of resistance phase (Figure 5b).

Analysis of the distribution of self-actualization indicators in the group of teachers with low (1), medium (2), high (3) levels of manifestation of the phase of emotional burnout "Exhaustion" confirms the second trend on the scale "Representation about human nature". Significant differences were found between low and medium levels $(* * * p<.001)$ (Figure $5 \mathrm{c}$ ).

The revealed tendency shows that both overstated and lowered indicators of self-actualization on the scale of "Self-esteem" promote development of a phase of Tension that is a threat of professional burnout of teachers. Overstated indicators of self-actualization on the scale "Orientation in time" can contribute to the exit from the phase of Resistance or, conversely, contribute to its development. Overstated indicators of self-actualization on the scale "Representation about human nature", as well as lowered lead to the stage of Exhaustion in burnout.

Correlation analysis revealed the presence of significant negative relationships (from $r=.2$ to $r$ $=.8, p \leq .01$ ) between the vast majority of different aspects of self-actualization with the following strategies: distancing (10 correlations), self-control (8 correlations), acceptance of responsibility (7 correlations); avoidance ( 3 correlations, $p \leq .01,1$ correlation, $p \leq .05$ ), confrontational coping (2 correlations, $p \leq .05,1$ correlation, $p \leq .01$ ), search for support ( 1 correlation, $p \leq .05,1$ correlation, $p \leq .01)$. However correlation analysis also revealed the presence of significant positive relationships between some aspects of self-actualisation and certain strategies of coping-behavior: the strategy of positive revaluation correlates with the indicator of self-actualization Representation about human nature $(r=0.4, p \leq .01)$ and Synergy $(r=0.3, p \leq .01)$; the strategy of planning a solution to the problem correlates with the indicator of self-actualization Self-acceptance $(r=.2, p$ $\leq .05)$. This fact suggests that certain aspects of self-actualization provoke a tension and the formation of coping behavior, which obviously should alleviate the stress determined by the desire for self-actualization.

The calculation of Spearman's rank correlation coefficient allowed to establish groups of connections between the duration of occupational stress factors (experience, age), phases of emotional burnout of teachers (tension, resistance, exhaustion), the formation of coping strategies and various aspects of self-actualization (Figure 6).

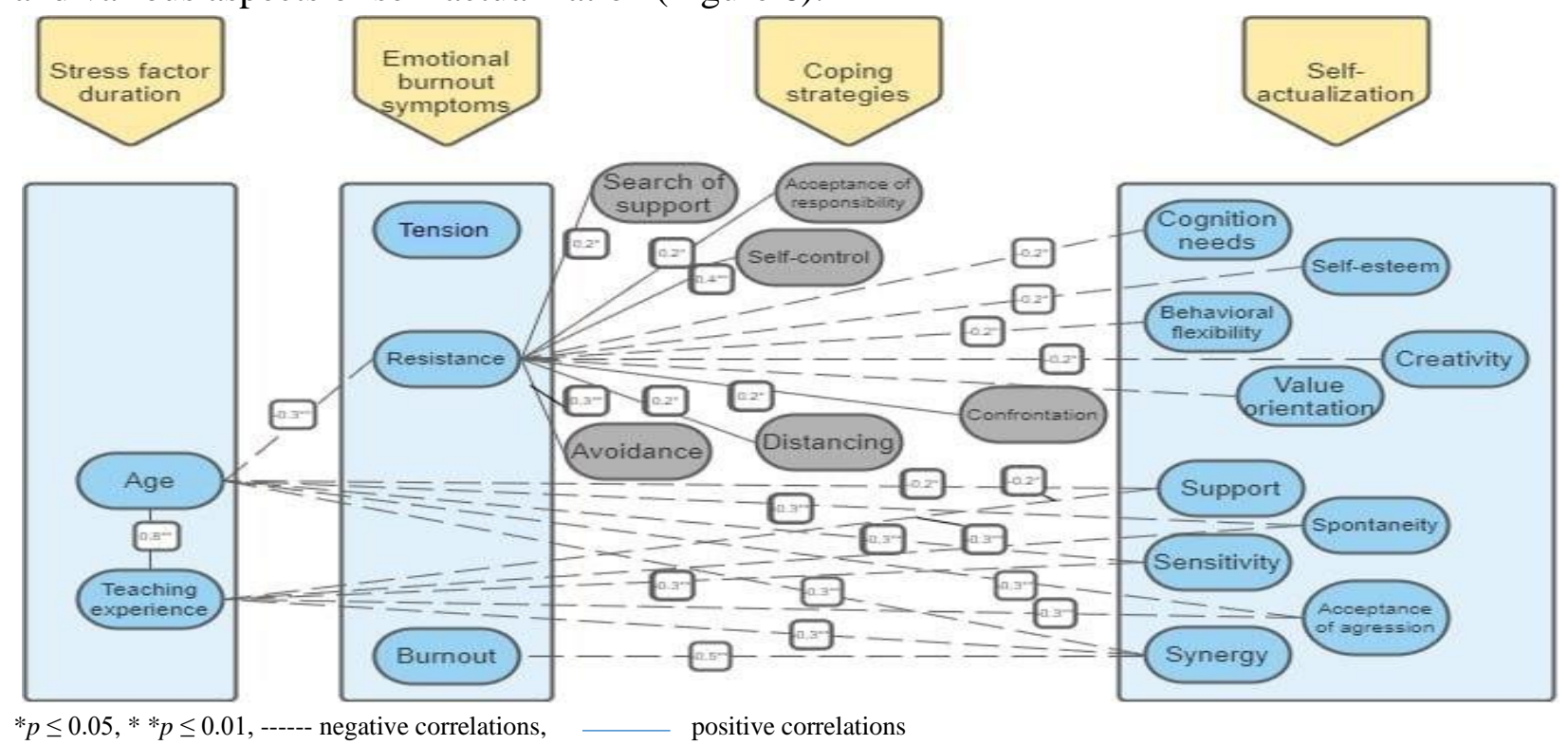

Figure 6. Correlations between variables 
The analysis revealed groups of correlations between the duration of occupational stress factors (experience, age), phases of emotional burnout (stress, resistance, exhaustion), the level of preference for certain coping strategies and indicators of self-actualization. The identified correlations can be interpreted as follows:

The presence of linear significant negative connections was found between the resistance phase in the process of emotional burnout and such aspects of self-actualization as Cognitive needs ( $r=-$ $.2, p \leq .05)$, Self-esteem $(r=-.2, p \leq .05)$, Creativity $(r=-.2, p \leq .05)$, Flexibility of behavior $(r=-$ $.2, p \leq .05)$, Value orientation $(r=-.3, p \leq .01)$. The phase of exhaustion in the process of emotional burnout is associated with one aspect of self-actualization Synergy $(r=-.5, p \leq .01)$. The presence of linear significant positive connections was found between the resistance phase in the process of emotional burnout and coping strategies such as search for support $(r=.2, p \leq .05)$, acceptance of responsibility $(r=.2, p \leq .05)$, self-control $(r=.4, p \leq .01)$, confrontational coping $(r=.2, p \leq .05)$, distancing $(r=.2, p \leq .05)$, and avoidance $(r=.3, p \leq .01)$.

Significant correlations were not found between the stress phase in process emotional burnout and other variables. Significant negative correlations of age with the resistance phase were found $(r=-.3, p \leq .01)$. This shows that many teachers with long experience, being in stressful conditions for many years, continue to work productively. But significant negative correlations were found between these indicators and certain aspects of self-actualization such as Synergy $(r=-.3, p \leq .01)$, Acceptance of aggression $(\mathrm{r}=-0.3, \mathrm{p} \leq 0.01)$, Sensitivity $(\mathrm{r}=-0.3, \mathrm{p} \leq 0.01)$, Spontaneity $(\mathrm{r}=-0.3$, $p \leq .01)$, Support $(r=-.2, p \leq .05)$. The obtained result obviously indicates a decrease in the need for self-actualization with increasing age and experience.

\section{Discussion}

This study focused on the nature of the relationship between self-actualization, occupational stress, and emotional burnout, taking into account the mediating effect of coping behavior. Analysis of the nature of the relationships between indicators of the level of self-actualization in groups of teachers with low, medium, high levels of development of each phase of emotional burnout allowed us to identify two trends.

The first trend suggests that a high level of certain aspects of self-actualization protects teachers from burnout, namely: significantly (eight self-actualization scales) delays the development of the tension phase, partially (two self-actualization scales) helps to overcome the resistance phase, plays a significant role (six self-actualization scales) in preventing the development of the phase of exhaustion in professional burnout of teachers. This result confirms the existing evidence in the literature that self-actualization is one of the most important ancillary resources of the individual, which prevents burnout (Babich, 2007; Nalichaeva, 2010). Previous research has shown that selfactualization causes productivity and satisfaction, growth of personal activity and the transition to a qualitatively new level of activity (Kurapova, 2009). We found a group of negative correlations between indicators of self-actualization and emotional burnout. The identified correlations confirm that self-actualization as a set of personality traits that make up the internal potential, determines the resistance of the individual to such negative psychophysiological states in professional activities as inadequate selective emotional response, emotional and moral disorientation (Nalichaeva, 2010). The ability of a person to holistically perceive the world around him slows down the development of the depletion phase. On the other side, according to a study by Bohan (2008), personal ambitions can also determine an occupational stress and deprive a person of resources. 
The second trend identified by us shows that overestimated or decreased indicators of some aspects of self-actualization are a threat to professional burnout of teachers. The analysis of correlations between variables also shows that certain aspects of self-actualization provoke tension and the formation of coping behavior, which, obviously, should alleviate the stress caused by the desire for self-actualization. We found the predominance of emotionally oriented coping strategies in the behavior of respondents. These results indicate a desire to reduce negative experiences, reduce stress through the exchange of feelings, thoughts and actions aimed at meeting the need to improve the emotional state of the individual in stress, maintain relationships with other people (Aripova, 2019). However, these strategies are assessed as less effective than problem-oriented coping behavior strategies (Lazarus \& Folkman, 1984; Rasskazova \& Gordeeva, 2011), so their application can cause an increase of stress. Thus, it can be concluded that self-actualization does not act as an independent mediator between occupational stress and emotional burnout. Predictors of successful professional adaptation are certain characteristics of coping behavior. In contrast to the study of Babich (2007), no significant correlation was found between the duration of stress and emotional burnout, except for age-related changes, which slightly reduce the ability to resist burnout. This shows that many teachers with many years of experience, being in stressful conditions for many years, continue to work productively.

\section{Conclusions and Future Study}

The pilot study confirmed the hypothesis that the nature of the link between emotional burnout as a result of occupational stressors and self-actualization may change due to the influence of some other variables, including coping behavior strategies. The study showed that a high level of selfactualization slows down the development of emotional burnout in teaching. The respondents who have optimal characteristics of self-actualization, the phase of resistance in the process of emotional burnout is less pronounced. However, overestimated and reduced indicators of certain aspects of self-actualization contribute to the development of the phase of tension and even the phase of exhaustion in emotional burnout. Overestimated self-actualization indicators are associated with the desire of subjects to create a better impression. Obviously, a person who is unable to realize their potential, feels additional stress. Thus, the obtained data confirm the existing ideas about selfactualization as a system-forming personal resource for overcoming burnout. The results also showed that self-actualization does not act as an independent mediator between occupational stress and emotional burnout. Certain aspects of self-actualization provoke tension and the formation of coping behavior, which, obviously, should alleviate the stress caused by the desire for selfactualization. Thus, the predictor of successful professional adaptation are certain characteristics of coping behavior. The advantage of emotionally-oriented coping strategies in the behavior of respondents was revealed, that indicates a desire to reduce negative experiences, reduce stress and improve emotional state. However, these strategies are assessed as less effective than problemoriented, which indicates that respondents do not have productive coping skills. Contrary to our expectations, no significant correlations were found between the duration of stress factors (experience) and emotional burnout, except for age changes, which slightly reduce the ability to resist burnout. This shows that many teachers with long experience, being in stressful conditions for many years, are able to work productively. The results also indicate a decrease in the need for self-actualization with increasing age and experience. 
Due to the limited sample, this conclusion is intermediate. Checking the identified trends for stability and reproducibility requires further research. The role of self-actualization in the regulation of professional burnout over a long period of time has been insufficiently studied. It seems also productive to identify the mediating role of certain coping strategies that have a significant impact on the success of professional adaptation in different organizational contexts.

\section{References}

Aripova, A. O. (2019). Emotional personality support as coping in stressful situations of various subjective complexity (Unpublished doctoral dissertation). Kostroma. Retrieved from https://www.dissercat.com/content/emotsionalnaya-podderzhka-lichnosti-kak-sovladanie-v-stressovykh-situatsiyakh-raznoisubekti

Babich, O. I. (2007). Personal resources for overcoming the burnout syndrome of teachers (Unpublished doctoral dissertation). Khabarovsk. Retrieved from https://www.dissercat.com/content/lichnostnye-resursy-preodoleniya-sindroma-professionalnogo-vygoraniya-pedagogov

Bagheri, J., Mirzaei Daryani, S., Sattari Ardabili, F., Azadi, B., \& Ahmadlu, M. (2019). The effects of management skills on job satisfaction at different organizational levels in banks: The mediating role of self-efficacy and social support. International Journal of Organizational Leadership, 8, $12-21$

Bityutskaya, E. V. (2015). Coping method questionnaire: a methodological guide. Moscow, Russia: IIU MGOU.

Bohan, T. G. (2008). Stress and stress tolerance: the experience of cultural-historical research. Tomsk: Ivan Fedorov Publishing House.

Boyko, V. V. (1996). Energy of emotions in communication: a view of oneself and others. Moscow, Russia: Filin.

de Almeida, M. H. (2019). Role stress, social support, job satisfaction. Behavior Studies in Organizations, 1, 2536. https://doi.org/10.32038/JBSO.2019.01.03

Fetiskin, N. P., Kozlov, V. V., \& Manuilov, G. M. (2002). Socio-psychological diagnostics of the development of personality and small groups. Moscow: Publishing house of the Institute of Psychotherapy.

Frydenberg, E. (2002). Beyond Coping. Meeting goals, visions and challenges. Oxford University Press.

Kurapova, I. A. (2009). Moral-value regulation of emotional burnout in professional activities (for example, teachers of secondary and higher schools) (Unpublished associate degree's thesis). Moscow. Retrieved from https://www.dissercat.com/content/nravstvenno-tsennostnaya-regulyatsiya-emotsionalnogo-vygoraniya-v-professionalnoideyatelnos

Lazarus, R. S. (1974). Psichological stress and coping in adaptation and illness. The International Journal of Psychiatry in Medicine, 5(4), 321-333.

Lazarus, $R$. (2006). Emotions and interpersonal relationships: toward a person-centered conceptualization of emotions and coping. Journal of Personality, 74(1), 9-43.

Lazarus, R., \& Folkman, S. (1984). Stress, appraisal and coping. Stress, appraisal, and coping. New York: Springer Pub. Co.

Losoya, S., Eisenberg, N., \& Fabes, R. (1998). Developmental issues in the study of coping. International Journal of Behavioral Development, 22(2), 287-313.

Maslach, C. M. (2003). Job burnout: new directions in research and intervention. Current Directions in Psychological Science, $12,189-192$.

Nalichaeva, S. A. (2010). Features of the professional burning out for teachers with the different level of self-realization. Scientific Bulletin of UNFU, 20(7), 321-329.

Rasskazova, E. I., \& Gordeeva, T. O. (2011). Coping strategies in the psychology of stress: approaches, methods, perspectives. Psikhologicheskie Issledovaniya, 3(17), 4.

Sattari Ardabili, F. (2020). Moderating-mediating effects of leader member exchange, self-efficacy and psychological empowerment on work outcomes among nurses. Organizacija, 53(3), 246-258. https://doi.org/10.2478/orga-2020-0016

Skaalvik, E. M., \& Skaalvik, S. (2015). Job satisfaction, stress and coping strategies in the teaching profession - What do teachers say? International Education Studies, 8, 181-192.

Voitenko, E. (2020). Emotional burnout as a result of professional stress in the work of managers. Journal of Behavior Studies in Organizations, 3, 5-12. https://doi.org/10.32038/JBSO.2020.03.02 